\title{
Skin Picking in Individuals with Intellectual Disability
}

\author{
Allison Cowan*, Jason Lee \\ Department of Psychiatry, Boonshoft School of Medicine, Wright State University, Dayton, USA \\ Email address: \\ allison.cowan@wright.edu (A. Cowan) \\ ${ }^{*}$ Corresponding author

\section{To cite this article:} \\ Allison Cowan, Jason Lee. Skin Picking in Individuals With Intellectual Disability. Clinical Neurology and Neuroscience. \\ Vol. 2, No. 1, 2018, pp. 9-11. doi: 10.11648/j.cnn.20180201.12
}

Received: August 5, 2017; Accepted: November 20, 2017; Published: January 9, 2018

\begin{abstract}
Individuals with Intellectual Disability seek treatment from primary care physicians, neurologists, and psychiatrists for excoriation disorder, a disorder described as the picking of ones' skin resulting in sores. There is a significant financial and emotional cost to people with this disorder. There is a lack of research in the area of co-occurring ID/D and excoriation disorder. This case series seeks to fill a gap in the literature by describing three distinct, successful treatments of excoriation disorder in individuals with intellectual disability.
\end{abstract}

Keywords: Excoriation Disorder, Intellectual Disability, Co-Occurring Mental Illness in ID/D, Skin-Picking Disorder in ID/D

\section{Introduction}

Excoriation disorder (ED), also known as skin-picking disorder, was previously categorized as an impulse-control disorder not otherwise specified [1]. In 2013 the DSM 5 described excoriation disorder as a discrete disorder [2]. The diagnostic criteria explain excoriation disorder as being on a continuum of obsessive compulsive disorders with a cycle of rising anxiety and a relief of tension with subsequent excoriation. This results in recurrent skin picking causing lesions, repeated attempts to decrease or stop skin-picking, and clinically significant distress or impairment in social, occupational, or other important areas of functioning. Exclusion criteria of ED are

1. Skin picking cannot be due to the physiological effects of a substance like cocaine or methamphetamine. These substances can cause individuals to have intense itching or causing sensations similar to bugs crawling under the skin.

2. ED cannot be due to another medical condition, like scabies infection or contact dermatitis. These conditions can cause intense itching resulting in excoriations.

3. It cannot be "better explained by symptoms of another mental disorder" [2]. Delusions can cause people to believe that they have parasites under their skin in Delusional Disorder, Somatic Type. Individuals with schizophrenia or other psychotic disorders can experience tactile hallucinations that can cause skin- picking. Body dysmorphic disorder is the belief that a perceived flaw in the body is debilitating. People with Autism or other causes of stereotypic movements can have symptoms causing self-injury.

Excoriation exists also as a part of the excessive grooming disorders like trichotillomania and onychophagia (nailbiting), where the tension-release cycle is relevant as well. In trichotillomania, the affected individual feels the urge to pull or pluck hair and experiences relief thereafter, and individuals with onychophagia describe the same tensionrelease cycle. There is some evidence that a genetic component exists as illustrated with mice models [3]. In these mice a genetic mutation caused them not only to groom themselves more but their littermates as well.

People with and without intellectual disability may describe the sensation as an urge to pick a spot of skin. There may be a visible defect, a bump, a scab, or clear skin. The resultant picking of the skin relieves this urge and provides a rewardthe release of dopamine and a reduction in anxiety or tension. People with intellectual disabilities may show an increased prevalence of this illness. The general population has a prevalence of $1-5 \%(4,5)$. Certain groups of individuals with Intellectual Disability have higher rates of ED as noted by Lang et al [6] including those with Smith-Magenis Syndrome, and Prader-Willi Syndrome. In a 2012 twin study, Monzani et al showed that genetic factors accounted for approximately $40 \%$ of variance [7]. There is scant evidence for treatment of 
individuals with intellectual disability and ED. However, the 2010 review by Lang et al of behavioral treatments reports that response interruption and redirection, differential reinforcement, protective clothing, punishment, and extinction were all helpful. While there remains little evidence of the pharmacological treatment of excoriation, several case reports demonstrate medications including risperidone, Nacetylcysteine, and topiramate can be helpful $[8,9,10]$.

Treating excoriation disorder with co-occurring ID/D can be difficult due to limitations in expressive communication. A person with ID/D can struggle with limited expressive language, in comprehension, and in having more people interpreting the actions of the individual. Patients with ID/D can have difficulty expressing the desire to reduce picking, and in the DM-ID-2 [11], this criterion is modified so as to no longer be required to make the diagnosis. Often patients' families and caregivers are more upset about the excoriations than are the patients themselves. However, the financial and emotional cost of these disorders can be significant with sores vulnerable to infection requiring hospital admissions and antibiotics. ED can impact work attendance, as many habilitation centers and sheltered workshops require skin to be intact without open sores. It can also impact recreation activities such as swimming or other sports for the same reasons. Part of the treatment for excoriation disorder includes a comprehensive workup for medical causes of scratching including liver failure, contact dermatitis (like poison ivy), or infectious diseases (like varicella). Patients can often present after years of struggling with these symptoms and with little hope for recovery, but as these cases demonstrate, there are treatments available that can be helpful.

The biopsychosocial model is a standard part of medical education. In treating excoriation disorder, care can be paid to each of these factors in both diagnosis and treatment. The Biological predisposition of ED has been linked to at least two separate variables and is more likely in those individuals with obsessive-compulsive disorder (OCD) and in first-degree family members than those in the general population [13]. Psychological factors include the stress-relief sensation and that often skin picking increased in times of increased emotional stress. Social factors can include the functional impact of the illness, e. g., social isolation and restrictions on recreation activities. Re-engagement in social activities, support, and education for direct care staff can be helpful in treatment.

\section{Three Cases}

\section{Case 1:}

Ms. A is a 35-year-old Asian American woman who has a diagnosis of Generalized Anxiety disorder and Severe Intellectual Disability. She lives in a group home. Upon exam, her physician noted a few open excoriations on her face, forearms, and stomach. Each was less than a centimeter with clustering of lesions. They were also noted to be of differing ages - some open, some with granulation tissue, and numerous small, circular scars. These findings are consistent with excoriation disorder. Her home care providers, case manager, and family reported that these sores worsened when Ms. A was suffering from increased interpersonal stressors. They reported she had recently been moved to a new area in her workshop after having been with her previous supervisor for close to 10 years. Her team reported that she was observed picking at her skin, was reminded not to pick, but was unable to stop excoriating. She underwent a thorough medical evaluation for other possible sources of pruritis with no other cause determined. She was started on sertraline, a selective serotonin reuptake inhibitor (SSRI), for anxiety and titrated to $200 \mathrm{mg}$ over the course of two months. The sertraline was effective in treating her anxiety but showed only modest benefit for excoriation symptoms. Ms. A had previously been prescribed a typical antipsychotic. Her family reported that when this medication was stopped some time ago, she experienced an increase in anxiety. Augmentation with low-dose quetiapine, an atypical antipsychotic medication, produced significant benefits of both anxiety and excoriation. She continues to be seen in follow-up and is stable on this treatment regimen. Her team helps her make healthy food choices and has increased her activities to address any possible weight gain with an atypical antipsychotic. To address her loss concerning her changed work environment, her direct care staff helped her write letters to her previous supervisor to maintain a connection.

Case 2:

Ms. B is a 55-year-old Caucasian woman presenting to her physician for treatment of agitation associated with autism spectrum disorder, with supports and moderate intellectual disability. She lives in a group home with 3 other individuals. Ms. B and her roommates are busy with many activities, which she enjoys. During the initial exam, the patient's home care provider, Ms. Z spoke to Ms. B in a child-like, demeaning manner. Ms. Z typically interrupted Ms. B to answer questions the physician asked of the patient. Ms. Z would also describe many insignificant as well as significant infractions of the rules of the house. Ms. Z would bring handwritten lists of the incidents describing Ms. B's emotion volatility and anger dysregulation. Ms. B would become visibly upset during the recounting of the incidents and pick at her skin. Ms. Z would then say, "See? She's doing it right now!" Ms. B had scarring from old excoriations on her scalp and arms. She had open lesions on her face and scalp. Due to these lesions, she was no longer able to participate in her swim recreational program, which was upsetting to Ms. B. She additionally felt embarrassed about her sores and worried that people looked at them. Ms. B met criteria for Excoriation disorder with recurrent skin picking which resulted in lesions, skin picking causing clinically significant social and occupational impairment, and her picking was not attributable to other illnesses or psychological effects of a substance. She was referred to a psychotherapist who began Response Prevention Therapy. The patient was taught to do deep pressure stimulation to her forearms when feeling the urge to pick. With prompting she was able to do this successfully. Education in combined sessions with Ms. B and Ms. Z together helped Ms. Z better understand Ms. B's condition. Education was provided to all the home care 
providers about appropriate interactions with people with intellectual disabilities. After a change of in-home care providers and in conjunction with the Response Prevention Therapy, Ms. B had significant improvement with excoriation as well as agitation. As of the last follow-up Ms. $\mathrm{B}$ had not had open excoriations for six months.

Case 3:

Mr. C is a 42-year-old African American male who has diagnoses of Down Syndrome, Moderate Intellectual Disability, and Delusional Disorder, erotomanic type. $\mathrm{He}$ believes that he is married to reality TV celebrity. Mr. C had a series of stressors including the accidental destruction of one his most prized possessions that he believed had come from his "wife." His family of origin was struggling with turmoil and isolation, and the absences from his sheltered workshop. He typically has an open lesion on his forehead at which he is seen picking. Coinciding with the increased stressors, the open excoriation became bigger and deeper. His family declined treatment with an antipsychotic medication, so Mr. C was titrated from N-acetylcysteine (NAC) $600 \mathrm{mg}$ twice a day to $1200 \mathrm{mg}$ twice a day and had $90 \%$ closure of the open area. His staff was trained in redirection, using competing stimuli, and acceptance training. At most recent follow-up, he had no open areas on his scalp and only a few around his cuticles and forearms. He reports that he is pleased with this treatment so that he can go back to his sheltered workshop job

$\mathrm{N}$-acetylcysteine, Response Prevention Therapy, and SSRI with atypical antipsychotic augmentation were found to be helpful. Additional mindfulness when attending to psychosocial stressors was also found to be impactful in choosing treatment approaches and is easily incorporated into the biopsychosocial formulation. Each of these patients had involved case management and care teams that were invested in recovery, which is likely also a beneficial factor.

\section{Conclusion}

These cases demonstrate that there are several ways to target comorbid illnesses and address ED as well. People with intellectual and developmental disabilities often can pose complicated clinical questions and are often omitted from the scientific literature due to being part a common exclusion criteria for studies. Multimodal treatments following the Biopsychosocial Model are helpful in maximizing therapeutic impact. Biological interventions include those medications described herein. Behavioral interventions including cognitive behavioral therapy, habit reversal training, and acceptance and commitment therapy have proven to be helpful $(6,11)$. Additionally, attention should also be paid to the environment in which the individual lives. Two of these three cases had significant social stressors that, after mitigation, provided a pronounced impact on care, and this final portion of the Biopsychosocial Model is too often neglected in the rush of medication management appointments. Advances in this newly defined diagnosis continue to be made, giving great hope to its successful treatment in the future. Some important clinical pearls in treating those with ED and intellectual disability include using the biopsychosocial model with diagnosis and treatment. Some medications may be of benefit include Nacetylcysteine, risperidone, and topiramate as well as selective serotonin re-uptake inhibitors. Finally, behavioral modifications including Habit Reversal Training, cognitive behavioral therapy, and acceptance and commitment therapy have proven effective in treating excoriation disorder.

\section{References}

[1] American Psychiatric Association. Diagnostic and statistical manual of mental disorders. Fourth edition. (DSM-IV-TR). American Psychiatric Pub, 2000.

[2] American Psychiatric Association. Diagnostic and statistical manual of mental disorders (DSM-5®). American Psychiatric Pub, 2013.

[3] Graybiel, Ann M., and Esen Saka. "A genetic basis for obsessive grooming." Neuron 33.1 (2002): 1-2.

[4] Keuthen, Nancy J., et al. "The prevalence of pathologic skin picking in US adults." Comprehensive psychiatry 51.2 (2010): 183-186.

[5] Hayes, Stephania L., Eric A. Storch, and Lissette Berlanga. "Skin picking behaviors: an examination of the prevalence and severity in a community sample." Journal of Anxiety Disorders 23.3 (2009): 314-319.

[6] Lang, Russell, et al. "Behavioral treatment of chronic skinpicking in individuals with developmental disabilities: A systematic review." Research in Developmental Disabilities 31.2 (2010): 304-315.

[7] Monzani, Benedetta, et al. "Prevalence and heritability of skin picking in an adult community sample: a twin study." American Journal of Medical Genetics Part B: Neuropsychiatric Genetics 159.5 (2012): 605-610.

[8] Roi, Cody, and Alessandra Bazzano. "Improvement in excoriation (skin-picking) with use of risperidone in a patient with developmental disability." Pediatric reports 9.1 (2017).

[9] Marler, Sarah, Kevin B. Sanders, and Jeremy Veenstra-Vander Weele. "N-acetylcysteine as treatment for self-injurious behavior in a child with autism." Journal of child and adolescent psychopharmacology 24.4 (2014): 231-234.

[10] Hamm, Brandon, Naveed Khokhar, and Xavier F. Jimenez. "Refractory Self-Injurious Behavior in Severe Intellectual Disability Responsive to Topiramate: A Case Report." Psychosomatics 58.2 (2017): 209-212.

[11] Fletcher, Robert Jonathan, Jarrett Barnhill, Sally-Ann Cooper. DM-ID-2: diagnostic manual-intellectual disability: a textbook of diagnosis of mental disorders in persons with intellectual disability. National Assn for the Dually Diagnosed, 2017.

[12] Stargell, N. A., Kress, V. E., Paylo, M. J., \& Zins, A. (2016). Excoriation Disorder: Assessment, Diagnosis and Treatment.

[13] Monzani, B., Rijsdijk, F., Harris, J., \& Mataix-Cols, D. (2014). The structure of genetic and environmental risk factors for dimensional representations of DSM-5 obsessivecompulsive spectrum disorders. JAMA psychiatry, 71 (2), 182-189. 